\title{
A Case of Susac Syndrome
}

\author{
Jonathan L. Zande, MS4', and \\ Muhammad U. Farooq, MD, FACP, FAHA ${ }^{2}$
}

(S)AGE

\author{
Keywords \\ Susac syndrome, cerebrovascular disorders, imaging, techniques, neuroimmunology, clinical specialty, neuroradiology
}

A 32-year-woman presented with mild encephalopathy, unsteady gait and instability, history of bilateral deafness, and bilateral visual changes. Magnetic resonance imaging of the brain demonstrated multiple hyperintensities involving the corpus callosum (Figure 1). There was no cord signal abnormality on imaging studies of the spinal cord. Imaging studies of the cervical and intracranial vasculature showed no evidence of vasculitis. Cerebrospinal fluid markers for inflammation and demyelination were negative, including oligoclonal bands. Visual evoked potentials were within normal limits. Extensive laboratory testing including Lyme antibody titers was unremarkable. Ophthalmology referral confirmed bilateral branch retinal artery occlusions. The patient was also referred for audiography evaluation, which confirmed bilateral hearing loss. These symptoms, retinal and audiographic examination, and imaging study findings were consistent with a diagnosis of Susac syndrome. Susac syndrome is an immune-mediated endotheliopathy characterized by the triad of encephalopathy, cochlear hearing loss, and retinal artery occlusions diagnosed by clinical examination supported by pathognomonic corpus callosum involvement on magnetic resonance imaging. ${ }^{1}$ The patient was initially treated with corticosteroids, but these were discontinued following resolution of symptoms, as Susac syndrome is a self-limiting condition. Should she experience recurrence of symptoms, options for therapy include a combination of immunosuppressive agents such as corticosteroids, intravenous immunoglobulin, methotrexate, mycophenolate, and cyclosphosphamide. ${ }^{2}$

\section{Declaration of Conflicting Interests}

The author declared no potential conflicts of interest with respect to the research, authorship, and/or publication of this article.

\section{Funding}

The author received no financial support for the research, authorship, and/or publication of this article.

\section{References}

1. Magro CM, Poe JC, Lubow M, Susac Jo. Susac syndrome: an organ-specific autoimmune endotheliopathy syndrome associated

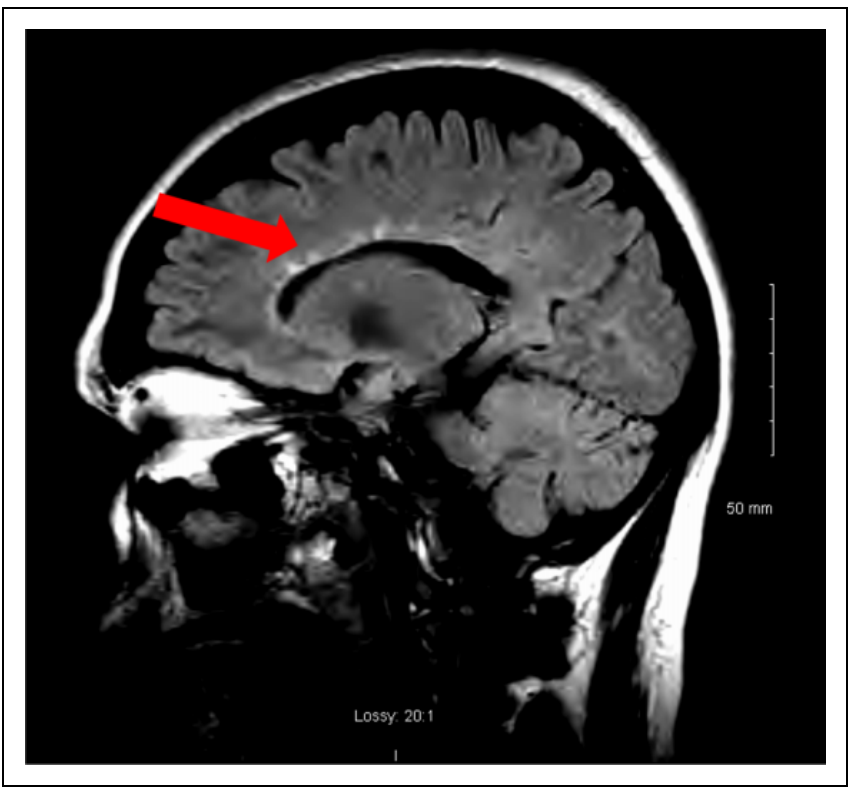

Figure I. Magnetic resonance imaging (MRI) of brain T2 fluidattenuated inverted recovery (FLAIR) demonstrating multiple hyperintensities involving the corpus callosum.

with anti-endothelial cell antibodies. Am J Clin Pathol. 2011; 136(6):903-912.

2. Garcia-Carrasco M, Mendoza-Pinto C, Cervera R. Diagnosis and classification of susac syndrome. Autoimmun Rev. 2014;13(4-5): 347-350. doi:10.1016/j.autrev.2014.01.038

'Michigan State University College of Human Medicine, Grand Rapids, MI, USA

${ }^{2}$ Division of Stroke and Vascular Neurology, Hauenstein Neuroscience Center, Saint Mary's Health Care, Grand Rapids, MI, USA

\section{Corresponding Author:}

Muhammad U. Farooq, Division of Stroke and Vascular Neurology Hauenstein Neuroscience Center, Saint Mary's Health Care, 200 Jefferson Ave SEGrand Rapids, MI 49503, USA.

Email: farooqmu@mercyhealth.com 